\title{
The variability of the amylose / amylopectin ratio and the preparative yield of Solanum tuberosum tuber starch
}

\author{
Y. Khoroshavin ${ }^{1,2 *}$, V. Khlestkin ${ }^{1,2}$ \\ ${ }^{1}$ Institute of Cytology and Genetics SB RAS, Novosibirsk, Russia \\ ${ }^{2}$ Novosibirsk State University, Novosibirsk, Russia \\ *e-mail:khoroshavin@bionet.nsc.ru
}

\section{Key words: potato, starch, physical and chemical properties, DNA markers}

Motivation and Aim: The optimal set of physicochemical properties of starch varies significantly for different applications and depends on the content and structure of its constituent polysaccharides: amylose and amylopectin. The content and structure of these molecules are regulated by the genes of their biosynthesis and can be considered as phenotypic features, according to which potato plants can be selected. Today many of these genes already known, but the connection of their allelic variants, loci with genes not yet described, and the physics-chemical characteristics of starch requires a deeper study. The purpose of this paper is to analyze the variability of amylose/amylopectin ratio as well as the content of potato starch and locating the «locus-feature» associations, which will subsequently allow to identify the markers of the corresponding genes.

Methods and Algorithms: The study was carried out on tubers of 90 varieties $S$. tuberosum from the collection "GenAgro", mainly blighty selection. Starch was isolated according to the procedure [1] and its preparative yield was calculated. To determine the amylose/amylopectin ratio, a new technique developed by us was used. Also DNA was isolated from the studied tubers and sent for genotyping to SNP chip of Illumina company [2]. Further, an analysis will be made of data on genetic variability of varieties and the finding of "locus-features" associations.

Results: We developed a new technique for measuring the ratio of amylose/amylopectin and obtained data for several dozen varieties of potato. Contrastive varieties (from 14 to $34 \%$ ) on the content of amylose in the starch the was detected. Data on the yield of starch for 90 varieties were also obtained and contrastive varieties (from 7 to $21 \%$ ) for this feature was found.

Conclusion: The data obtained show a significant variability in the features of tuber starch, and, consequently, the possibility of breeding on them. These data will be used to find the DNA markers of the relevant features.

Acknowledgements: Supported by the RFBR (17-29-08006).

\section{References}

1. Khlestkin V.K., Erst T.V. (2017) A practical guide to the starch granules' morphology study by microscopy. Vavilovskii Zhurnal Genetiki i Selektsii = Vavilov Journal of Genetics and Breeding. 21(6):728-734. DOI 10.18699/VJ17.290 (in Russian).

2. Vos P.G. et al. (2015) Development and analysis of a 20K SNP array for potato (Solanum tuberosum): an insight into the breeding history. Theoretical Applied Genetics. 128(12):2387-2401. DOI 10.1007/ s00122-015-2593-y. 